\title{
Profiling application potential for alkali treated sisal fiber-polypropylene composite in Ashby material-property plots
}

\author{
N. Mokaloba \\ Botswana Bureau of Standards, Engineering Testing Laboratories, Gaborone, BOTSWANA \\ E-mail: mokaloba@bobstandards.bw; tel. +267 3903200; fax. +2673903120
}

\begin{abstract}
The effect of alkali treatment on sisal fiber from the plant agave sisalana in appropriation for composite material application is presented. Effectiveness of the fiber's reinforcement potential within polypropylene (PP) matrix is evaluated through morphological analysis, crystallinity levels, and tensile, where ultimate tensile strength (UTS) of $580 \mathrm{MPa}$ are obtained. The performance property of the resultant composite is comparatively profiled against equivalent material systems in Ashby material property charts, where suitability for light, low-flexure and stiffness limited design applications is evident. Based on the fiber's non-uniform and irregular geometrical variation, the structural characteristics of the fiber is described by the two-parameter Weibull statistical technique, applied as control to ascertain experimentally determined parameters. The fiber diameters ranged from $148 \mu \mathrm{m}$ to $304 \mu \mathrm{m}$ and exhibited corbel-arch and horse-shoe morphology. The composite is of a randomly oriented shortfiber architecture, 0.23 fiber volume fraction. This study concludes that drawbacks to realizing functional cellulosic fiberpolymeric composites can be achieved by the judicious integrated choice of (i) methodology of fiber modification, (ii) proper fiber characterization, and (iii) optimization of processing parameters.
\end{abstract}

Keywords: Ashby plot, Weibull analysis, sisal-fiber composite, alkali treatment.

DOI: http://dx.doi.org/10.4314/ijest.v9i3.4

\section{Introduction}

Presently, there is an ever-increasing demand for newer, stronger, stiffer, and yet lighter-weight materials in the fields such as aerospace and automobile. The array of advanced materials includes polymer composites, nanocomposites, and biocompatible materials (Bledzki et al., 2008; Sarwar, 2010).High demands on materials for better overall performance has led to extensive research and development efforts in the composites fields as they offer properties superior in strength and modulus to many traditional engineering materials such as metals. As a result, these materials are now being rapidly utilized in industries than traditionally used metals, and they have become the forefront of research and development activity (Gupta et al., 2012; Lu et al., 2013). Natural fibers like sisal, jute, kenaf and coir have been used as reinforcement material in polymer-based composites. The incorporation of stiff fibers and soft matrices can lead to new materials with outstanding mechanical properties encompassing the advantages of both the fiber and matrix (Carvallo et al, 2010). The matrix transmits applied loads to the reinforcing fibers within the composite, resulting in a material with improved mechanical properties compared to the un-reinforced matrix material (Beckermann, 2007).

Sisal, being a source of lingocellulosic fiber, the composition of which includes cellulose, hemicelluloses, lignin, pectin, fat, waxes, and water soluble substances bear hydroxyl groups from cellulose and lignin (Anandjiwala et al., 2008). Owing to the presence of hydroxyl groups in cellulosic fibres, the moisture regain is high, leading to poor organic wettability with the matrix material and hence a weak interfacial bond between the reinforcing agent and hydrophobic matrices. The most important factor in obtaining mechanically-viable composite material is the fiber-matrix interfacial interaction, the extent of which depends upon the chemical structure and polarity of these materials (Zhaoqhian et al., 2011). 
In order to develop cellulosic fiber-polymeric matrix composites with better mechanical properties, it is necessary to increase the hydrophobicity of the reinforcing agent. Several chemical treatments exist such as alkalization, acetylation, liquid ammonia, graft polymerization and peroxides which are conducted on cellulosic fibers to modify the physical and mechanical properties (Anandjiwala et al., 2008). Favaro et al., 2010 investigated the effects of mercerization and acetylation on sisal fiber compatibility with oxidized high-density polyethylene and reported improved fiber-matrix adhesion. Liu et al., 2007 also reported improved fiber-matrix adhesion of mercerized and maleic anhydride grafted jute fiber reinforced polypropylene composites. Changes to the cellulose structure and crystallinity index were also reported by Jayaramudu et al., 2009 on mercerized sterculia urens fabric.

In this study, the cellulosic fiber sisal has been modified with alkali solution to impart viable physical and mechanical properties which make it suitable for application in composites.Notwithstanding the well-established observation of cellulosic fiber's geometric irregularity, this study considers a two-parameter Weibull statistical technique to describe the structural characteristics of the fiber. Weibull is appliedas control to ascertain experimentally observed parameters used for calculating UTS.

The structural constituents of the fiber being cellulose, hemicellulose and lignin are sensitive to the different ranges of temperatures. Kabir et al. (2011) reported degradation of lignin at $\sim 200{ }^{\circ} \mathrm{C}$, and hemicellulose at even higher temperatures. Kifani et al. (1996) reported hemicellulose to degrade between $220-315{ }^{\circ} \mathrm{C}$ and lignin between $280-500{ }^{\circ} \mathrm{C}$. Despite the advantages mentioned above, the use of cellulosic fibers in thermoplastics has had challenges associated with it. The possible reasons that contribute to unsatisfactory final properties of the composite are (i) limited thermal stability at typical melt processing temperatures of $\sim 200^{\circ} \mathrm{C}$, which limits the type of thermoplastic that can be used with the fibers, (ii) poor dispersion characteristics in the non-polar olefinic thermoplastic melt due to strong hydrogen forces between the fibers, and (iii) high moisture absorption of the fibers which can affect the dimensional stability of the composite (Kaushik et al., 2010; Li et al., 2011). Other researchers (Oladele et al., 2010; Nirmal et al., 2011) are in agreement that the limitation of cellulose fibers as reinforcement in thermoplastics shows that the aforementioned problems are common, independent of the type and origin of the fiber employed. The preceding challenges call for extensive research, technology development and innovation to affirm certainty in product development processes and predictable performance.

Compared to continuous-fiber composites, short-fiber composites can be easily processed in a similar manner to the matrix. Cellulose fibers are flexible and will not fracture when processed over sharp curvatures which enables the fibers to maintain the desired aspect ratio for good performance. Several researchers have suggested that an aspect ratio in the range of 100-200 after processing is essential for high performance of short-fiber composites (Kabir et al., 2011; Li et al., 2008). On the other hand, Joseph et al. (1993) reported maximum tensile strength properties based on the aspect ratio of 20, when studying sisal-fiber polyethylene composites. The volume fraction also, has profound influence on fiber-matrix interface failure and is one of the parameters used in determination of natural fiber composite mechanical properties (Lu et al., 2013). The composite used in this study was prepared from mercerized sisal fiber at volume fraction, $V_{f}$ of 0.23 , at length of $5 \mathrm{~mm}$. This accorded better fiberpacking, less fiber-entanglement, and ease of dispersion within the PP matrix. The fiber used showed comparatively superior UTS of $588 \mathrm{MPa}$, percentage crystallinity of $59.8 \%$, and $13 \%$ thermal improvement effect compared to other alkali treatment protocols employed. To account for material anisotropy and random fiber-orientation architecture, composite strength was estimated from the modified "Rule of Mixtures". The model is represented by the relationship (Lu, 2002; Li et al., 2000; Xiaolin, 2008)

$$
\sigma_{c}=\sigma_{m} V_{m}+\sigma_{f} V_{f}(x)
$$

where $\sigma_{c}$ represents the strength of the composite, $\sigma_{m}$ and $\sigma_{f}$ are stresses in the matrix and fiber correspondingly, and $V_{m}$ and $V_{f}$ are the matrix and fiber volume fractions correspondingly. The parameter $x$ determines the strength transfer between fiber and matrix.

El-Messiry (2012) studied tensile properties of okra, sisal and banana fibers and epoxy composites and observed optimal mechanical properties of the composite at $V_{f}$ of $25 \%$. Li et al. (2000) studied properties of sisal fiber reinforced polystyrene and observed initial reduction in tensile strength at $V_{f}$ of $10 \%$ followed by optimal properties at $V_{f}$ of $20 \%$.Various mathematical models, namely, Halpin-Tsai, Hirch's, Bowyer-Bader consider randomly oriented short-fiber composite strength to be influenced by (i) fiber length, (ii) fiber volume fraction, (iii) fiber orientation, (iii) fiber dispersion, and (iv) fiber-matrix interfacial strength $(\mathrm{Lu}, 2002)$.This research contributes to the present challenges which scientists and engineers face in seeking solutions to realizing improved fiber-matrix adhesion characteristics of plant-based fibers and polymeric matrices for the benefit of the ever increasing application needs. The objective of this study is to evaluate effects of alkali treatment on sisal fiber for application in composite, identify potential causal to degradation of the fiber structural integrity, and subsequently assign market application potential of the resultant composite from comparative evaluation of its engineering properties with equivalent material-systems using Ashby plots.

\section{Materials}

Sisal fibers used in this study were harvested in locally. The ribbon fibers were separated mechanically from the inner core of the sisal leaf. Laboratory reagents used were sodium hydroxide pellets of $99 \%$ strength supplied by Rochelle Chemicals, South Africa. Chemicals were diluted to $6 \%(\mathrm{w} / \mathrm{w})$ solution concentration. The polypropylene polymer used as matrix was supplied by Sasol Polymers, Republic of South Africa. 


\section{Experimental Techniques}

3.1 Determination of fiber geometrical properties: The fiber diameter was measured using the Motic digital microscope (DM143) interfaced to the Motic image PLUS 2 computer software for automated image analysis. Fifty fibers were randomly selected and the dimensional parameters, namely, diameter, perimeter and cross-sectional area measured. To control and ascertain the accuracy of diameter of the fiber specimen, the two-parameter Weibull statistical technique was used. For measurement, the fiber was held into position on a recessed card-board which exposed the geometry to be measured. As for diameter, three equidistant measurements were taken along the length of the fiber and the mean reported.

3.2Alkali treatment protocol: The fibers were soaked in sodium hydroxide $(\mathrm{NaOH})$ of $6 \%(\mathrm{w} / \mathrm{w})$ solution treatment for $30 \mathrm{sec}, 1 \mathrm{hr}$ and $3 \mathrm{hr}$ duration. Samples were coded Al-6-30s, Al-6-1hr, Al-6-3hr, each representing immersion times and the common $6 \%$ solution concentration. Following immersion, fibers were washed with distilled water and dried at room temperature for $48 \mathrm{hr}$. The fibers were then soaked in $1 \%$ acetic acid to neutralize excess sodium hydroxide. The fibers were then rinsed with distilled water and dried in an oven at $80^{\circ} \mathrm{C}$ for $2 \mathrm{hr}$ to remove free water.

3.3 Fiber crystallinity: The changes in fiber crystallinity were evaluated using X-ray diffraction. The experiments were performed at ambient condition using X-Pert PRO mpd control diffractometer using $\mathrm{Cu} \mathrm{K \alpha}(1.5405980 \AA)$ radiation Ni-filter and scintillation counter as detector at $45 \mathrm{kV}$ and $30 \mathrm{~mA}$. All the experiments were performed in the continuous mode at a scan speed of $4^{\circ}$ per minute and a step size of $0.05^{\circ}$. The X-ray diffractograms were recorded from 0 to $70^{\circ}$ of $2 \theta$ (Bragg angle). The area method was used to evaluate fiber crystallinity. The percentage crystallinity was determined using Equation 2, where I (002) is the counter reading at peak intensity at a $2 \theta$ angle close to $22^{\circ}$ (crystalline material) and $\mathrm{I}_{(\mathrm{am})}$ is the amorphous counter reading at a $2 \theta$ angle of about $18^{\circ}$ (Kumar et al., 2017).

$$
\% C r=\left\{I_{(002)} /\left(I_{(002)}+I_{(a m)}\right)\right\} x 100
$$

3.4 Thermo-gravimetric analysis (TGA): TGA was used to study the thermal stability of the fiber following alkali treatment. The Pyris 1 Thermo-gravimetric analyzer supplied by PerkinElmer was used in the analysis. Samples measuring $6.7 \mathrm{mg}$ for untreated fiber, and $6.9 \mathrm{mg}$ for the Al-6-1-hr treated fibers were analysed. The samples were held for $1 \mathrm{~min}$ at $30{ }^{\circ} \mathrm{C}$ and then heated from 30 ${ }^{\circ} \mathrm{C}$ to $600{ }^{\circ} \mathrm{C}$ at $10{ }^{\circ} \mathrm{C} / \mathrm{min}$ in nitrogen atmosphere flowing at $20 \mathrm{ml} / \mathrm{min}$.

3.5 Surface morphology: Surface morphology was used to study effects of alkali treatment on the surface structures of untreated and treated sisal fibers. The samples were analysed using the scanning electron microscope Model XL 30. Excitation energy was set at 12-15 keV, with analysis at $10 \mathrm{~mm}$ working distance. Fiber conductivity was via carbon deposition.

3.6 Tensile testing: Tensile testing for fibers was conducted according to ASTM D3379-75 using the Zwick/Roell Z020 universal testing machine. A test speed of $2 \mathrm{~mm} / \mathrm{min}$ was used and a gauge length of $50 \mathrm{~mm}$ was maintained. The fiber cross-sectional area was calculated using diameter measured from the Motic Images Plus 2.0 ML software. Average strength values for five specimens from the Al-6-30s,Al-6-1 hr andAl-6-3hr treated fibers were reported. Tensile test for the composite was conducted according to the ISO 527-1 standard, using the Zwick/Roell Z020 Universal tensile tester. A crosshead speed of $3 \mathrm{~mm} / \mathrm{min}$ and gauge length of $50 \mathrm{~mm}$ were maintained. The average strength values for five specimens from the Al-6-1hr treated fiber composite were reported.

3.7 Preparation of the composite: The sisal-PP blend was achieved by melt-mixing. The sisal fiber was added to a melt of polypropylene and mixing was performed in a Haake Rheocord mixer at $180{ }^{\circ} \mathrm{C}$, rotor speed of $30 \mathrm{rpm}$, for a period of 10 minutes. The composite panels were prepared through compression moulding using the Wabash mould press. A pressure of 0.5 metric tons was applied and kept constant throughout the compression cycle. Figure 1 shows the resultant composite panel, which exhibits darkening effect and fiber agglomeration. Tensile test specimens were prepared from the fabricated composite panels which showed no fiber agglomeration.

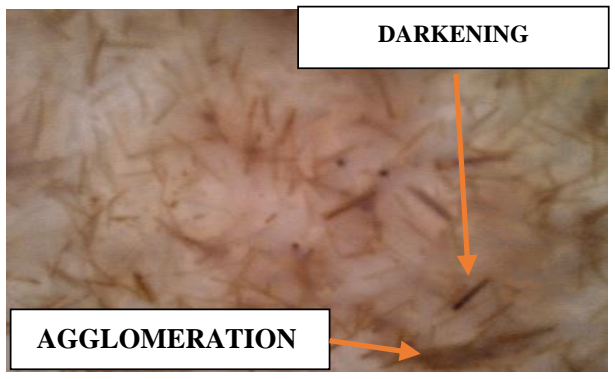

Figure 1. Composite panel showing fiber darkening and agglomeration. 


\section{Results and Discussions}

4.1Fiber geometrical characteristics: The fiber cross sections were neither circular nor fairly uniform as shown by the SEM micrographs in Figure 2(a) and (b). The fiber-end shapes observed were the horse-shoe and the corbel-arch types.

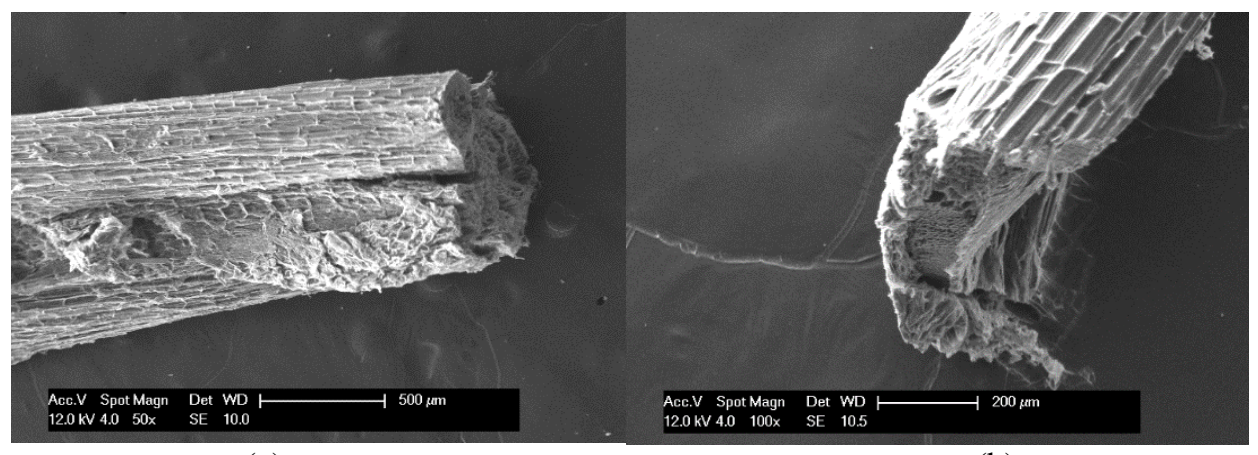

(a)

(b)

Figure 2. Fiber-end shapes depicting the (a) horse-shoe and the (b) corbel-arch type

A wide dimensional variation was recorded which confirmed the geometrical non-uniformity of the sisal fiber. It was observed that $48 \%$ of the fiber diameters lay between $150 \mu \mathrm{m}-225 \mu \mathrm{m}$ and $10 \%$ of the fiber diameters lay between $300 \mu \mathrm{m}-375 \mu \mathrm{m}$.

For applied two-parameter Weibull model, the cumulative failure probability $F(t)$ is given by the relationship

$$
F(t)=1-\exp \left[-\left(\frac{1}{t_{o}}\right)^{m}\right]
$$

where $t$ is the dimension, $m$ is the Weibull shape parameter and $t_{o}$ is the scale parameter. The parameter $m$ is a measure of the variability of the fiber dimensions, where a large value of $m$ corresponds to small scatter in dimension. The scale parameter $t_{o}$ is the measure of statistical dispersion of the data.

The cumulative probability $P_{i}$, under a particular dimension is approximated by:

$$
P_{i}=\left(n_{i}-0.5\right) / n
$$

where $n_{i}$ is the number of fibers having dimensions at or below a certain value and $n$ is the total number of fibers measured (Awal et al., 2003).

A plot of $\ln \left[-\ln (1-P) v s \ln (t)\right.$ was used to determine the shape parameter $m$ and scale parametert $t_{o}$ for the diameter, perimeter, and cross-sectional area as shown in Figure 3(a) to (c) correspondingly. The parameters $m$ and $t_{o}$ were taken as the gradient and the $y$-intercept of the best fit line correspondingly (Awal et al., 2003).The shape parameter for diameter was the largest at 3.03 vs 2.22 and 0.99 for perimeter and cross-sectional area correspondingly. This translates to a smallest dimensional scatter for diameter, which as consequence, was used to calculate fiber UTS.

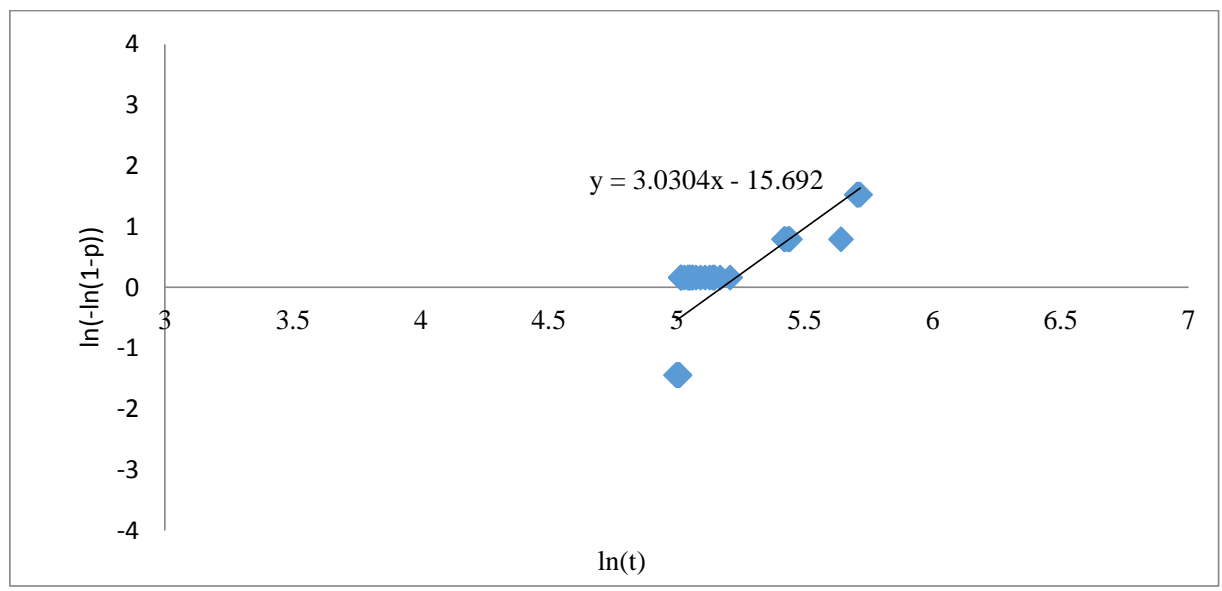

(a)Diameter. 


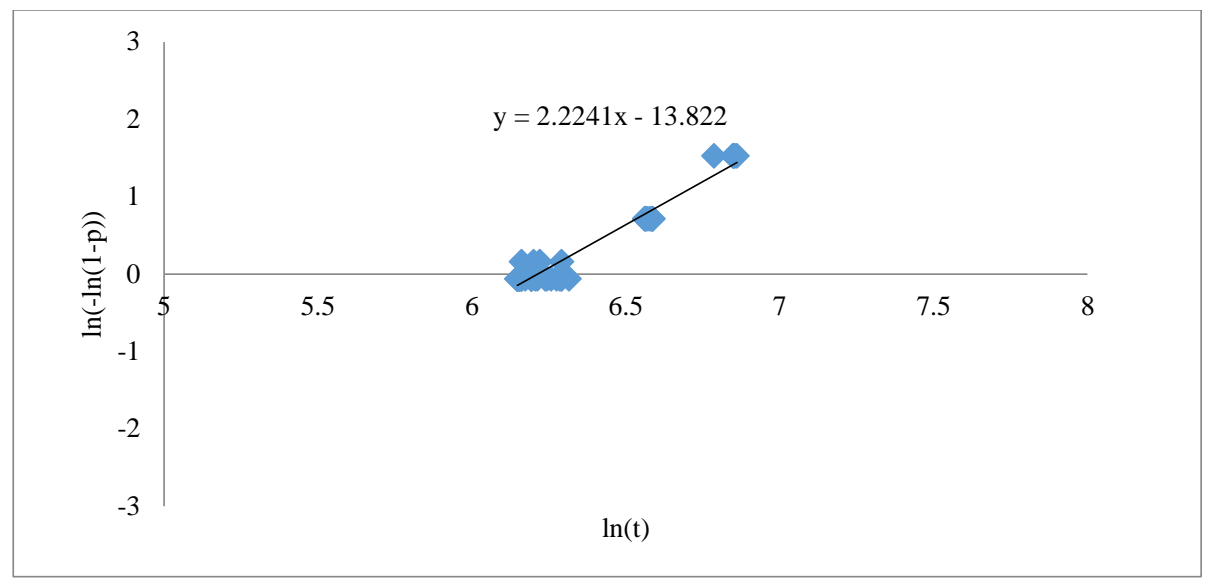

(b)Perimeter.

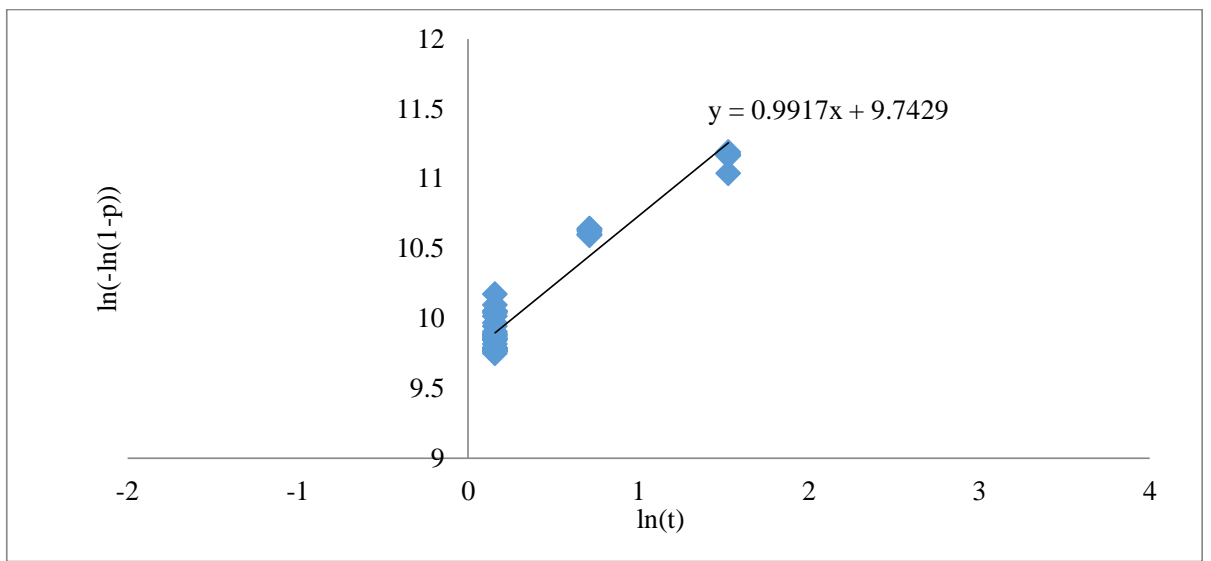

(c)Cross sectional area.

Figure 3. Shape and scale parameter plots for fiber geometrical characteristics.

4.2Morphology analysis

4.2.1 Helical structure morphology: Figure 4 shows the micrograph of a cellulose helical spiral with intermittent transverse fractures sites shown by an arrow. The occurrence of fracture is attributed to excessive mechanical forces sustained during decortication, as the fiber is liberated from the pithy core of the sisal leaf. Susceptibility of cellulose helical spiral fracturing has also been reported by Anandjiwala et al., 2008, who indicated spiral fractures in coir fibers following decortication. The figure shows coil fractures having occurred as far apart as $\sim 80 \mu \mathrm{m}$. The transverse fractures on the spiral ranged from minor splits to complete circumferential dissociation of adjoining coil as shown by the insert, on the same figure.

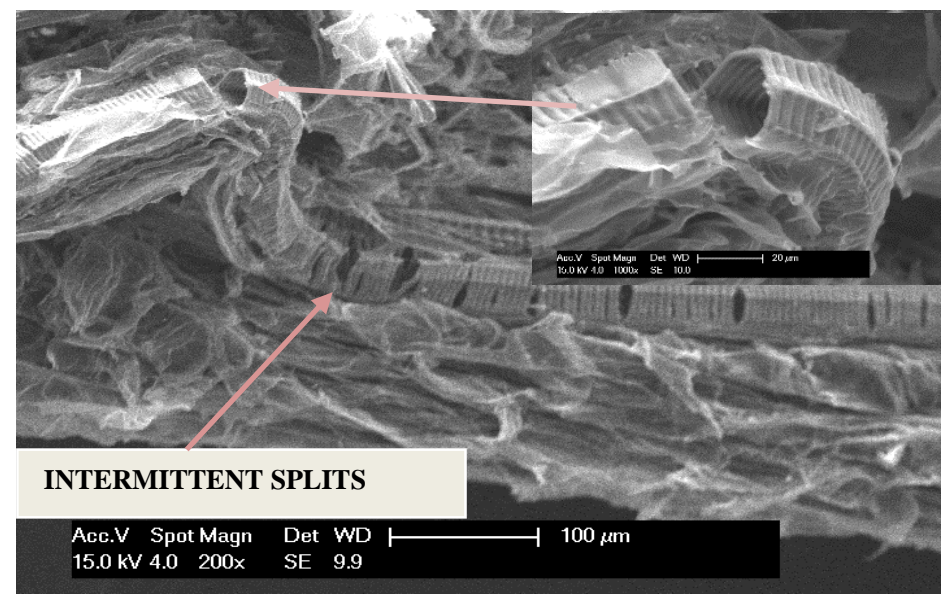

Figure 4.Helical spiral structure depicting intermittent splits. Enlarged insert depicts the six-sided structural geometry. 
4.2.2Fiber pull-out surface morphology: Figure 4(a) shows a relatively smooth resin socket from which an untreated fiber was pulled out. The micrograph indicates poor interfacial bonding due to incompatibility or poor surface wetting of hydrophilic fiber and hydrophobic matrix. Figure 4(b) shows a peripheral perspective of a resin socket from which the 6-Na-1hr treated fiber was pulled out. Fiber microfibrills attached to the periphery of the socket (indicated by the arrows) indicates better interfacial adhesion due to improved roughness, wettability and impregnation of matrix

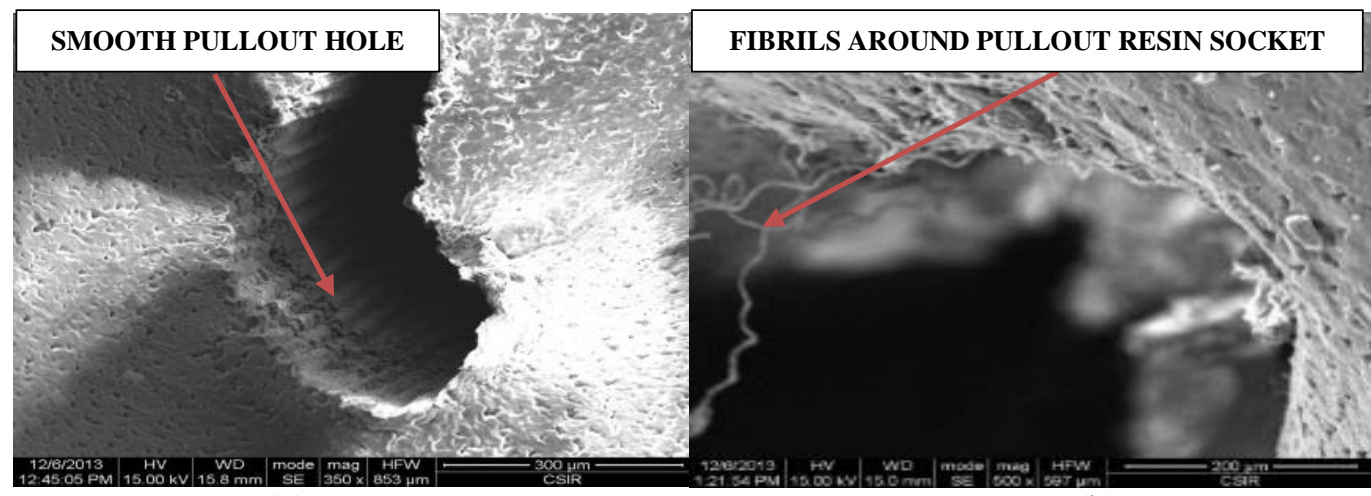

(a)

(b)

Figure 5. Resin sockets from (a) untreated fiber, (b) Al-6-1hr treated composites after fiber pullout

Figures 6(a) and (b) show the SEM micrographs of the longitudinal sections of the pullout holes depicting comparative morphologies for counter surfaces from which the Al-6-1hr treated fiber was pulled out. From the figures, the sparsely distributed pits (rough terrain) on matrix surfaces (ranging from $18.5 \mu \mathrm{m}$ to $65 \mu \mathrm{m}$ size) surrounding the fiber pull-out region can be observed. They can be considered to point towards need for improvement in composite fabrication or the nature of the matrix.

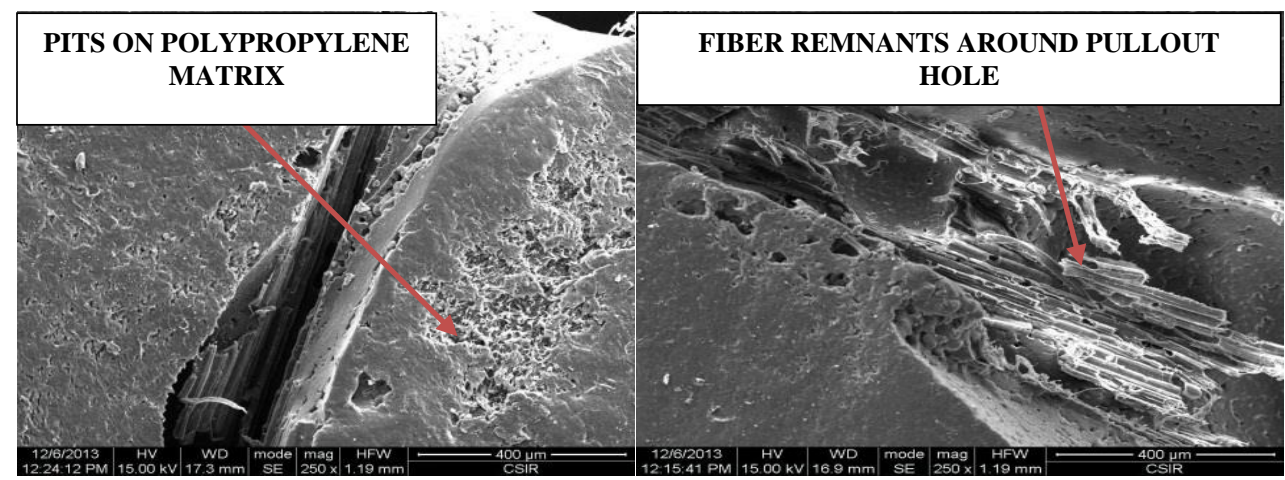

(a)

(b)

Figure 6. Counter surfaces from which Al-6-1hr treated fibers were pulled out.

4.3 Fiber crystallinity: The diffractographs were used to calculate percentage crystallinity for untreated and treated fibers. Improved crystallinity facilitates optimal packing of cellulose crystallites, a condition with potential to effect improved fiber stiffness, which is advantageous for composite application (Mwaikambo et al. 1999). The percentage crystallinity for the untreated sisal fiber was used as the baseline index for comparative evaluation of the effect of crystallite packing in the fiber caused by treatment. The peak intensities and percentage crystallinity calculated from diffractographs are presented in Table 1 for the untreated fibers, and the Al-6-30sec, Al-6-1hr, and Al-6-3hrtreated fibers. Samples treated for $1 \mathrm{hr}$ and $3 \mathrm{hr}$ showed the same percentage crystallinity of approximately $13 \%$ improvement compared to the untreated fiber.

Table 1. Peak intensities and corresponding percentage crystallinity for fibers.

\begin{tabular}{|l|l|l|l|}
\hline Sample & $\mathrm{I}_{18}$ & $\mathrm{I}_{22}$ & $\% \mathrm{Cr}$ \\
\hline Untreated & 650 & 700 & 51.8 \\
\hline Al-6-30sec & 4300 & 5700 & 57.0 \\
\hline Al-6-1hr & 3400 & 6400 & 59.8 \\
\hline Al-6-3hr & 3400 & 6400 & 59.8 \\
\hline
\end{tabular}

4.4 Thermogravimetric analysis (TGA): Table 2 presents the thermal degradation parameters for untreated and Al-6-1hr fibers determined from TGA thermograms. The results indicate the initial degradation for the Al-6-1 hr fibers to have improved from 174 ${ }^{\circ} \mathrm{C}$ to $230{ }^{\circ} \mathrm{C}$, reflecting a $56{ }^{\circ} \mathrm{C}$ shift when compared to the untreated fiber. This phenomenon confirms that the Al-6-1hr treated fiber could be processed at temperatures lower than $230{ }^{\circ} \mathrm{C}$ whilst maintaining its structural integrity. A similar viable shift was 
observed for the inflection point (where degradation rate is maximum) and the final degradation temperature. The results are consistent with findings by Kifani et al. (1996) who observed degradation of hemicellulose to lie between $220{ }^{\circ} \mathrm{C}-315^{\circ} \mathrm{C}$, cellulose to degrade at about $370{ }^{\circ} \mathrm{C}$, and lignin between $280{ }^{\circ} \mathrm{C}-500{ }^{\circ} \mathrm{C}$

Table 2. Thermal degradation parameters for untreated and Al-6-1-hr treated fiber.

\begin{tabular}{|l|l|l|}
\hline Parameter & Untreated fiber & $\mathrm{Al}-6-1 \mathrm{hr}$ \\
\hline Initial degradation & $174^{\circ} \mathrm{C}$ & $230^{\circ} \mathrm{C}$ \\
\hline Inflection point & $335^{\circ} \mathrm{C}$ & $379^{\circ} \mathrm{C}$ \\
\hline Final degradation & $425^{\circ} \mathrm{C}$ & $475^{\circ} \mathrm{C}$ \\
\hline
\end{tabular}

4.5 Tensile test results for the fiber: Table 3 shows the average values for the ultimate tensile strength (UTS), range of tensile moduli, strain at failure for the untreated and treated fibers. Untreated fibers showed average UTS value of $440 \mathrm{MPa}$ and a tensile modulus range of $6.99-8.26 \mathrm{GPa}$. The highest UTS for the treated fiber $588 \mathrm{MPa}$ for Al-6-1hr, which showed a $33.6 \%$ improvement compared to the untreated fiber. The Al-6-1 hr treated fibers also, exhibited a corresponding superior improvement in percentage crystallinity of $59.8 \%$.The improvement in tensile modulus exhibited by alkali treated fibers is attributed to improved stiffness imparted by chemical treatment and has been reported by other researchers (Kaushik et al., 2010).

Table 3. Tensile properties for untreated and treated fibers

\begin{tabular}{|l|l|l|}
\hline Fiber & UTS $(\mathrm{MPa})$ & Tensile modulus $(\mathrm{GPa})$ \\
\hline Untreated & 440 & $6.99-8.26$ \\
\hline Al-6-30s & 473 & $20.50-21.68$ \\
\cline { 1 - 2 } Al-6-1hr & 588 & \\
\hline Al-6-3hr & 554 & \\
\hline
\end{tabular}

4.6 Tensile test results for the Composite:Table 4 shows UTS and tensile modulus results for the untreated fiber composite and the Al-6-1hr fiber composite. The UTS for the Al-6-1hr fiber composite improved by $20.5 \%$ in average, when compared to the composite from the untreated fiber. The tensile modulus for the Al-6-1hr composite also increased which suggests enhanced fiber reinforcement potential from surface treatment. A similar observation of improvement in tensile strength for composites prepared from chemically treated fibers has been reported by several researchers (Zhaoqian et al., 2011; Ghasemi et al., 2010). Zhaoqian et al. (2011) reported improvement of $6.77 \%$ in tensile strength for graft co-polymerized sisal fiber in polylactide composites.

Table 4.Experimental tensile properties of untreated and Al-6-1hr composite, $V_{f}$ of $23 \%$.

\begin{tabular}{|l|l|l|}
\hline Material type & UTS (MPa) & Tensile Modulus (MPa) \\
\hline \multirow{5}{*}{ Untreated fiber composite } & 34.3 & 202 \\
\cline { 2 - 3 } & 36.4 & 218 \\
\cline { 2 - 3 } & 31.9 & 208 \\
\cline { 2 - 3 } & 37.2 & 164 \\
\cline { 2 - 3 } & 35.6 & 189 \\
\cline { 2 - 3 } & Av 35.1 & 196 \\
\hline \multirow{5}{*}{ Al-6-1hr fiber composite } & 41.7 & 230 \\
\cline { 2 - 3 } & 44.1 & 235 \\
\cline { 2 - 3 } & 39.9 & 235 \\
\cline { 2 - 3 } & 40.6 & 228 \\
\cline { 2 - 3 } & 45.0 & 231 \\
\cline { 2 - 3 } & $\mathrm{Av}$ & 232 \\
\hline
\end{tabular}

From Equation 1, the UTS values approximates to $\sigma_{c}=44.7 \mathrm{MPa}$, where the parameter $x$, which accounts for material anisotropy and random short-fiber orientation architecture is approximated to 1/5(Xiaolin, 2008). The theoretical prediction of UTS is observed to be higher than the average experimental value i.e., $44.7 \mathrm{MPa} v s 42.3 \mathrm{MPa}$ by $5.8 \%$.This effect is attributed to a combination of the following factors:

The microstructure of the composite, shown from Figure 6, which depict surface irregularities in a form of pits of $\sim 18.5 \mu \mathrm{m}$ to 65 $\mu \mathrm{m}$ approximate sizes. The surface pits lead to cracks which act as stress concentration points for crack initiation and failure propagation of the composite during service.

The architectural variables, particularly the aspect ratio and fiber dispersion. Figure 1 and Figure 4 depicts fiber agglomeration and helical structure splits correspondingly, being the possible effects with potential to weaken composite strength. 


\subsection{Profiling Composite properties in Ashby plots}

The comparative analysis of mechanical properties of the Al-6-1hr treated fiber composite against other materials systems by means of the Ashby plots is presented. Comparative visualization of performance characteristics is made against composites made from reinforcement materials such glass fiber and natural fibers including polypropylene-based composites.

4.7.1 Young's modulus vs. Density comparison: Figure 7 shows the Ashby plot for the Young's modulus vs Density. The combination of the Young's modulus and density allows for design of light and stiff products, where high values of specific stiffness indicate good materials for lightweight, stiffness-limited design (Ashby et al., 1995). From the figure, it can be observed that Young's modulus for engineering polymers range from $0.07 \mathrm{GPa}-10.2 \mathrm{GPa}$ and the density ranges from $\sim 0.38$ to $2.4 \mathrm{Mgm}^{-3}$. The Al-6-1hr fiber composite (tensile modulus: $0.232 \mathrm{GPa}$; Density: $1120 \mathrm{~kg} / \mathrm{m}^{3}$ ) shown by a star symbol, lay within the envelope for engineering polymers. The guide lines $\frac{E^{\frac{1}{3}}}{\rho}=C, E / \rho=c$ and $\frac{E^{\frac{1}{2}}}{\rho}=C$ represent material performance indices and they have a slope of unity i.e., have the same specific stiffness (Ashby et al., 1995).

For stiffness guideline on performance of light and stiff materials (a component loaded in bending) $\frac{E^{\frac{1}{2}}}{\rho}$ or for flat plates in bending $\frac{E^{\frac{1}{3}}}{\rho}$, the Al-6-1hr fiber composite suits potential for materials of light, low elastic deformation and moderate bending applications.

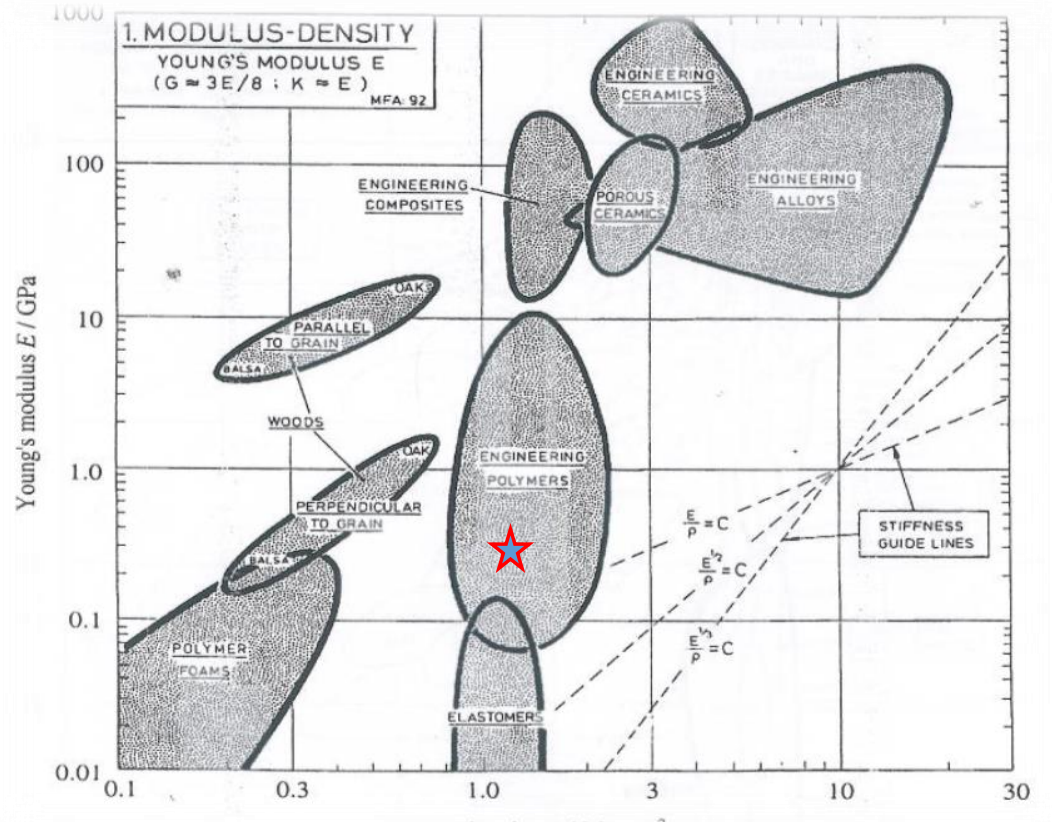

Figure 7. Young's modulus vs. Density (Ashby property chart applied with permission: Ashby et al., 1995).

4.7.2 Tensile strength vs. Density for the Composite: Figure 8 shows the Ashby plot for UTS vs Density. The Al-6-1hr treated sisal fiber polypropylene composite with UTS of $42.3 \mathrm{MPa}$, and $\rho=1120 \mathrm{~kg} / \mathrm{m}^{3}$, shown by a star symbol, lay at the lower strength domain within the envelope for 'natural polymers and polymer composites. The guidelines $\frac{\sigma_{f}^{\frac{2}{3}}}{\rho}$ and $\frac{\sigma_{f}^{\frac{1}{2}}}{\rho}$ depicts loading for a plate material in flexure (Ashby et al., 1995). This identifies potential for a structurally efficient, light and strong material system, with strong panels selected for high values of $\frac{\sigma_{f}^{\frac{1}{2}}}{\rho}$. The Al-6-1hr fiber composite suits the domain for low flexural strength applications. 


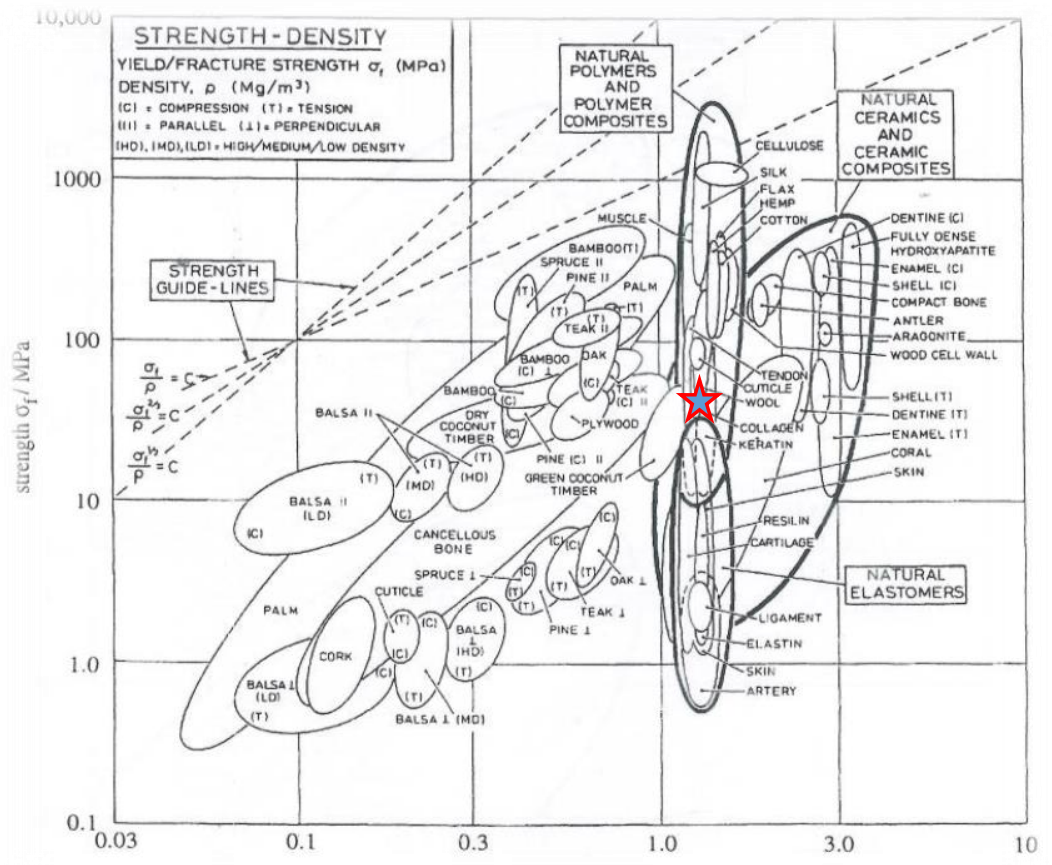

Figure 8. Tensile strength $v s$. Density (Ashby property chart applied with permission: Ashby et al., 1995).

\section{Conclusions}

On the overall, the resultant composite exhibits beneficial comparative functionalities based on composite strength, fiber integrity in terms of improved percentage crystallinity and tensile properties and manufacturing attributes from improved fiber morphology. The following conclusions are reached on theprofiled composite's market application potential.

- The Ashby profiling of the Al-6-1hr treated composite exhibits a material system suitable for light, low-stiffness applications such as automobile interior panels/linings and profiles.

- The cellulose helical spirals aresusceptible to fractures from mechanical stresses sustained from extraction. Usage of suitable decortication forces during fiber liberation from the leaf's pithy core is underscored.

- From the fiber's geometric irregularity, the Weibull statistical technique can effectively describe fiber structural characteristics and ascertain accuracy of parameters used in characterization of natural fibers.

\section{Acknowledgements}

The author expresses his sincere gratitude to the African Materials Science and Engineering Network (AMSEN), University of Botswana node for financial support towards this research.

\section{References}

Alavadeen A., Rajin N., Karthikegan S., 2015. Mechanical properties of banana/kenaf fiber reinforced hybrid polyester composites: Effect of woven fabric and random orientation, Materials and Design, Vol. 66, pp. 246-257.

Anandjiwala D. R., John M. J., 2008. Recent Developments in Chemical Modification and Characterization of Natural FiberReinforced Composites, Wiley InterScience, Society of Plastics Engineers, pp. 188-207.

Awal A., Cescutti G.,Ghosh S.B.,Mussig J., 2003). Interfacial studies of natural fiber polypropylene composites using single fiber fragmentation test, Composite Part A, Vol. 42, pp. 50-56.

Bledzki A. K., Mamun A. A., Lucka-Gabor M., Gutowski V. S., 2008. The effects of acetylation on properties of flax fiber and its polypropylene composites, eXPRESS Polymer Letters, Vol. 2, No. 6, pp. 413-422.

Favaro S. L., Ganzerli T.A., De Carvalho, Neto A. G. V., Da Silva O. R. R. F., Radovanovic E.,2010. Chemical, morphological and mechanical analysis of sisal fiber-reinforced recycled high-density polyethylene composites,

eXPRESS Polymer Letters, Vol. 4, No. 8, pp. 465-473. Gerard T. Pott, 2004. Natural fibers with low moisture sensitivity. Kluver Academic Publishers. ISBS 14020784 36, USA, pp. 155-210. 
Jayaramudu J., Guduri B. R. and Rajulu, A. V., 2009, Characterization of natural fabric SterculiaUrens, International Journal of Polymer Analysis and Characterization, Vol. 14, No. 2, pp. 115-125.

Kaushik K. Vijay, Kumar A., Vashistha S., and Kalia S., 2010. Effect of chemical treatment on the morphology, structure and properties of sisal fibers, International Conference on Advances in Polymer Technology, India, pp. 248-252.

Kozlowski R., 2004. Composites from natural fibers and composites, Kluwer Academic Publishers, ISBS 14020784 36, USA, pp. 255-266.

Kumar R., Sharma S., Pathak D., Arora N., 2017. Ionic conductivity, FTIR and thermal studies of nanocomposite plasticized proton conducting polymer electrolytes. Solid State Ionics, Vol. 305, pp. 57-62.

Li Y, Chunjing H., Yehong Y., 2008. Interfacial Studies on sisal fiber reinforced high density polyethylene composites. Composite Part A, Vol. 39, pp. 570-578.

Liu X. Y., Dai G. C., 2007. Surface modification and micromechanical properties of jute fiber mat reinforced polypropylene composites, eXPRESS Polymer Letters, Vol. 1, No. 5, pp. 299-307.

Lu N., Shubhashini O., 2013. Thermal stability and thermo-mechanical properties of hemp - high density polyethylene composites: effect of two different chemical modifications, Composites, Part B, Vol. 44, pp. 484-490.

Moigne N. L., Longery M., Taulemesse J. M., Benezet J. C., Bergeret A., 2014. Study on the Interface in natural fibers reinforced poly(lactic acid) bio composites modified by optimised organosilane treatments. Industrial Crops and Products, Vol. 52, pp. 481-494.

Mwaikambo, L. Y., Ansell, M. P., 1999. Effect of chemical treatment on the properties of hemp, sisal, jute, and kapok fibers for composites reinforcement. $2^{\text {nd }}$ International Wood and Natural Fiber Composites Symposium, Kassel/Germany, pp. 1-16.

Newaz G. M, 1993. Polymer-matrix Composites, Materials Science and Technology, Vol. 13, VCH, New York, USA.

Ngaowthong C., Ringsadthorng V., Siengchin S., 2016. Polypropylene/hemp woody core fiber composites: morphology, mechanical, thermal properties and water absorption behaviours. Advances in Mechanical Engineering, Vol. 8, No. 3, pp. 1-10.

Nirmal U., Singh N., Hashim J., Saijod T.W. L., Jamil Nadia., 2011. On the effect of different polymer matrix and fiber treat ment on single fiber pullout test using betelnut fibers. Materials and Design, Vol. 32, pp. 2717-2726.

Ouajai S., Hodzic A., Shanks R. A., 2004. Morphological and grafting modification of natural cellulosic fibers, Journal of Applied Polymer Science, Vol. 94, pp. 2456-2465.

Torres F. G., Cubillas M. F., 2005. Study on the interfacial properties of natural fiber reinforced polyethylene, Polymer Testing, Vol. 24, pp. 694-698.

Wong K. J., Yousif B. F., Low K. O., 2009. The effects of alkali treatment on the interfacial adhesion of bamboo Fibers. Materials: Design and Applications, Proceedings, IMechE Part L, Vol. 224, pp. 139-147.

Yan L., Yiu-Wing M., Lin Y., 2000. Sisal fiber and its composites: a review of recent developments. Composites Science and Technology, Vol. 60, pp. 2037-2055.

\section{Biographical notes}

N. Mokaloba holds a B. Eng.in Mechanical Engineering from University of Botswana (1998), and an MSc in Chemical Engineering (2006) from University of Pretoria, where his study interests were in polymer science, polymer processing, and polymer engineering. He holds a PhD (Engineering) from the Faculty of Engineering \& Technology, University of Botswana (2016), where his research was on development of development of polymer-based composite material system. His research area was on optimization of cellulosic-fiber properties and characterization of the resultant composite with estimation of elastic properties using finite element analysis. He has published articles in the broad area of optimization of cellulosic fibers and their engineering application. He has presented at conferences in the enunciated scientific area.He is presently a test engineer (engineering materials) with Botswana Bureau of Standards, where he has been responsible for quality evaluation of engineering materials and products for the past 17 years, with inclination to the polymeric.

Received April 2017

Accepted May 2017

Final acceptance in revised form June 2017 